\title{
El aprendizaje servicio como experiencia en el Grado de Educación Primaria
}

\author{
Antonio Martínez \\ Universidad de Almería, España
}

\section{Resumen}

La universidad, como institución social de referencia, es cada vez más consciente de que su función no se puede realizar al margen de la responsabilidad social que desempeña. El modelo formativo universitario actual requiere situaciones de aprendizaje que permitan combinar aprendizaje académico y formación para una ciudadanía activa. Desde este planteamiento, se presenta una experiencia de aprendizaje servicio realizada en una asignatura en el título de Grado en Educación Primaria, cuyo objetivo es servir como estrategia metodológica en la formación de una ciudadanía crítica entre los futuros maestros. Su puesta en práctica nos ha permitido conocer las diferentes actitudes del alumnado hacia la intervención social, favoreciendo el contacto con diversas instituciones del entorno a través de la colaboración en sus programas, realizando diferentes tipos de servicio acorde a sus aprendizajes e intereses.

\section{Palabras clave}

Aprendizaje servicio, experiencia, educación primaria. 


\title{
Service learning as experience in the Degree of Primary Education
}

\begin{abstract}
The university, as a social institution of reference, is increasingly aware that its function can not be carried out regardless of the social responsibility it plays. The current university training model requires learning situations that allow combining academic learning and training for active citizenship. From this approach, a service learning experience is presented in a subject in the Degree in Primary Education, which aims to serve as a methodological strategy in the formation of a critical citizenship among future teachers. Its implementation has allowed us to know the different attitudes of students towards social intervention, favoring contact with various institutions of the environment through collaboration in their programs, performing different types of service according to their learning and interests.
\end{abstract}

\section{Keywords}

Service-learning, experience, primary education. 


\section{Introducción}

En un contexto sociocultural cada vez más complejo, la institución universitaria tiene un papel crucial contribuyendo al aprendizaje de conocimientos especializados y habilidades técnicas de actuación relacionadas con la introducción de valores de responsabilidad social tanto a nivel individual como colectivo (Arranz, 2011). En este caso, hacemos referencia a un ejercicio de la responsabilidad que no se limita únicamente a la rendición de cuentas, sino de carácter ético, es decir, un modelo formativo de universidad que apueste de manera decidida por alcanzar una mayor inclusión social, la formación de titulados orientados al logro del bien común y a la consecución de una sociedad que sea más justa y democrática. En este sentido, consideramos que la universidad debe ser un referente en la transmisión de principios éticos y valores para la ciudadanía.

El modelo formativo y de aprendizaje actual de la institución universitaria está orientado al logro de competencias. El objetivo fundamental es proporcionar una formación integral de los titulados, que incluya una formación deontológica en el ejercicio de las profesiones, una formación ciudadana y cívica que favorezca su inclusión social, así como una formación humana desde una perspectiva ética y moral. En este contexto, uno de los objetivos de la universidad debe centrarse en la promoción de una ciudadanía activa conectada con los contextos de aprendizaje, convivencia y formación mediante la introducción de competencias de carácter ético y social (Tejada, 2013). De ahí la necesidad de considerar su cometido en la generación de competencias relacionadas con el desarrollo humano que permitan la construcción personal y la convivencia social (Inciarte, ParraSandobal y Bozo, 2010).

Los actuales planes docentes y programas institucionales de las universidades apuestan por incorporar el logro de competencias transversales de carácter ético y social a través de contextos de aprendizaje propios de cada titulación. El objetivo fundamental es que el estudiante universitario participe activamente en la sociedad y que esté comprometido con ésta. Para ello es necesario proporcionar oportunidades educativas que faciliten los aprendizajes a través de experiencias en entornos reales. Las propuestas de aprendizaje servicio son un buen ejemplo de ello, favoreciendo el desarrollo de la educación para la ciudadanía entre los estudiantes universitarios (Puig, Batlle, Bosch y Palos, 2006; Tapia, 2006).

Un proyecto de aprendizaje servicio constituye una práctica compleja en la que se establece una estrecha vinculación entre el servicio a la comunidad y el aprendizaje, en una actividad articulada, coordinada y coherente (Puig et al., 2006). Las propuestas de aprendizaje servicio se pueden definir como una práctica que se realiza en la comunidad como respuesta a las necesidades sentidas y reales, que requiere de una planificación tanto de los contenidos curriculares como de las actividades a 
realizar y que promueve la participación de los estudiantes en las diferentes fases del proyecto (Montes, Tapia y Yaber, 2011).

A nivel metodológico, el aprendizaje servicio es una estrategia desarrollada a través de la investigación-acción que une el aprendizaje con el compromiso social y que tienen por objetivo la mejora de algún aspecto de la sociedad impulsando la participación ciudadana en la búsqueda del bien común. Como señalan Saz y Ramo (2015), los proyectos de aprendizaje servicio destacan la optimización de la calidad del aprendizaje académico del alumnado junto con la formación integral como personas socialmente responsables.

Las propuestas de aprendizaje servicio debe cumplir una serie de condiciones de manera que permitan desarrollar competencias transversales relacionadas con la dimensión social de la persona: toma de decisiones, trabajo en equipo, incorporación al tejido social, convivencia y dominio de diferentes lenguajes y de nuevas tecnologías (Martínez, 2006). En primer lugar, deben facilitar la reflexión sobre cuestiones sociales y éticamente relevantes para mejorar la comprensión crítica de los estudiantes. En segundo lugar, deben establecerse relaciones interpersonales entre los estudiantes y los profesionales de las entidades en un ambiente de reconocimiento y respeto mutuo. En tercer lugar, las prácticas deben organizarse mediante un trabajo cooperativo y colaborativo que permitan la reflexión individual. En cuarto lugar, proporcionar un aprendizaje práctico para la vida en comunidad mediante la formación en valores éticos y sociales. En quinto lugar, deben ser prácticas que puedan ser evaluadas contando con la participación de los propios estudiantes, los miembros de la comunidad y el profesorado responsable del proyecto.

\section{Descripción de la experiencia}

El propósito de la experiencia que se presenta a continuación es acercar el aprendizaje servicio a los estudiantes universitarios. Los objetivos específicos que se persiguen son promover actitudes en los estudiantes hacia la intervención social, favorecer el contacto con el tejido social del entorno a través del aprendizaje servicio y realizar servicios a la comunidad, acorde a sus intereses, vinculados al desarrollo de las competencias propias de la titulación.

La experiencia se inscribe dentro de la asignatura de formación básica impartida durante el primer curso del título de Grado de Educación Primaria de la Universidad de Almería denominada Sociedad, Escuela y Democracia. Tiene una carga lectiva de seis créditos ECTS. La ratio del grupo docente es de setenta estudiantes, repartidos en dos grupos de trabajo práctico que, a su vez, se organizan en equipos reducidos de cuatro o cinco estudiantes.

Entre las competencias que se trabajan en la asignatura, se incorporan tanto aprendizajes transversales relacionados con la competencia social y ciudadanía global que persiguen que los estudiantes muestren un compromiso 
con la realidad social, la participación en proyectos colectivos y la implicación en la realización del servicio; como con relacionar la educación con el medio y colaborar con las familias y la comunidad, que permiten al alumnado conocer la complejidad y riqueza del contexto comunitario, así como fomentar la participación con las familias y entidades del entorno.

Dicha experiencia se puso en marcha durante el curso académico 2015-2016. Su diseño y desarrollo se ha realizado con la participación conjunta del alumnado, profesorado y entidades sociales del entorno. Para esta experiencia se ha contado con diferentes entidades sociales de la provincia de Almería. Como actividad previa, se contactó con una serie de entidades del entorno social que tenían la necesidad de recibir un servicio a la vez que nos facilitan un campo de aprendizaje para los estudiantes universitarios. Durante las primeras sesiones de clase, estas entidades exponían el análisis social del barrio, su problemática y necesidades, se debatía, y se comenzaba a idear conjuntamente el proyecto de ApS. Se trata de un proyecto con un enfoque explícito de justicia social, entendida de forma bidimensional: una relacionada con la práctica y la otra con la reflexión (Aramburuzabala, 2013).

Desde la práctica, las acciones se dirigen a realizar labores de apoyo educativo a niños y a niñas en situación de desventaja social. El alumnado universitario se implicaba semanalmente en diversas actividades programadas que llevaban a cabo en la sede de la asociación elegida. Se pretende favorecer la igualdad de oportunidades, el éxito educativo y la convivencia, ofreciendo una educación intercultural ya que la mayor parte del colectivo atendido es inmigrante.

Desde la reflexión, los debates críticos giraban en torno a la educación en valores democráticos y participativos, los derechos humanos, las propuestas educativas de ApS y comunidades de aprendizaje, así como las desigualdades sociales por clase social, género y etnia, favoreciendo una ciudadanía reflexiva, comprometida con el cambio y la mejora social.

Para el diseño y desarrollo del proyecto ApS se ha seguido una serie fases: preparación, planificación, realización y seguimiento, evaluación multifocal y cierre (Palos, 2015).

En una primera fase se ha realizado el diseño del proyecto en dos etapas bien diferenciadas. La primera de ellas, la de preparación, en la que se abordan los aspectos relacionados con la ideación del proyecto, la identificación de las necesidades sociales y educativas, así como de las entidades sociales, centros educativos y apoyos institucionales con los que se iba a contar. A continuación, durante la etapa de planificación, se concretaron los diferentes elementos pedagógicos (objetivos generales y específicos, contenidos vinculados a la asignatura, lactividades a realizar, así como criterios de evaluación). Para completar esta etapa, en una segunda fase, se especificaron los elementos organizativos y de gestión, incluyendo un cronograma con las diferentes actividades programadas. 
En la segunda fase nos adentramos en el desarrollo del proyecto con la etapa de realización y seguimiento. En ella, el alumnado asiste semanalmente a la entidad social para llevar a cabo el desarrollo de lo planificado. En la fase de seguimiento se empleó, entre otros, la realización semanal de un diario reflexivo. Se parte de un esquema previo en el que se pide que recojan la descripción de las diferentes actividades realizadas en relación al servicio y a los aprendizajes, el análisis de los mismos e incidentes críticos, y una valoración personal con las observaciones que estimen oportunas. El profesorado ofrecía un feedback que le ayudaba a mejorar.

La fase de evaluación del proyecto se realiza mediante la aplicación de la Rúbrica de autoevaluación y mejora de los proyectos de ApS elaborada por el Grup de Recerca en Educació Moral (2014). Por último, se realiza una representación gráfica de los resultados que permite obtener una imagen clara de los aspectos fuertes y débiles del proyecto, estableciendo un punto de partida para elaborar las propuestas de mejora realizables a corto y medio plazo del proyecto.

Para la fase de cierre, los grupos de trabajo de estudiantes han realizado un informe en el que se ha realizado una evaluación final del proyecto que nos ha permitido recoger los resultados finales, realizar una visión de conjunto sobre lo realizado, incluyendo las reflexiones y una toma de decisiones para posibles actuaciones futuras. Asimismo, ha supuesto un reconocimiento a los logros obtenidos desde un punto de vista personal, grupal y comunitario.

\section{Resultados}

Como se ha descrito anteriormente, se aplicó una rúbrica de evaluación para el análisis del proyecto. Se analizaron siete dinamismos que fueron evaluados de manera conjunta por los participantes. En la tabla 1 se representan los resultados alcanzados durante el proyecto tanto en los dinamismos básicos (necesidades, servicio, sentido del servicio y aprendizaje) como en los pedagógicos (participación, trabajo en grupo y reflexión).

A cada uno de los dinamismos se le asignó una calificación que iba de uno al cuatro, siendo (1) el de menor implicación hasta (4) que corresponde a una mayor participación. Cada miembro del grupo establecía una calificación de manera personal que posteriormente era debatida en grupo con la intención de llegar a un consenso en cuanto a la valoración.

Tabla 1. Resultados de la aplicación de la rúbrica de evaluación

de evaluación
\begin{tabular}{|l|l|l|l|l|}
\hline DINAMISMOS & I & II & III & IV \\
\hline Necesidades & Ignoradas & Presentadas & Decididas & Descubiertas \\
\hline Servicio & Simple & Continuado & Complejo & Creativo \\
\hline $\begin{array}{l}\text { Sentido del } \\
\text { servicio }\end{array}$ & Tangencial & Necesario & Cívico & Transformador \\
\hline Aprendizaje & Espontáneo & Planificado & Útil & Innovador \\
\hline Participación & Cerrada & Delimitada & Compartida & Liderada \\
\hline $\begin{array}{l}\text { Trabajo en } \\
\text { grupo }\end{array}$ & Indeterminado & Colaborativo & Cooperativo & Expansivo \\
\hline Reflexión & Difusa & Puntual & Continua & Productiva \\
\hline
\end{tabular}

Fuente: Elaboración propia

\section{Conclusiones}

A partir del análisis de la rúbrica de evaluación sobre los dinamismos del proyecto, podemos concluir cuáles son los puntos fuertes y débiles del 
proyecto.

En cuanto a los puntos fuertes de la experiencia, destacar que el sentido del servicio responde a una necesidad cívica de la comunidad en la que los participantes han sido conscientes de la dimensión social del proyecto. El aprendizaje obtenido ha sido útil ya que las actividad formativas realizadas tienen una estrecha relación con el servicio, otorgando mayor calidad a la intervención. La reflexión ha sido continuada durante realización de todo el proyecto. En este sentido el diario reflexivo y crítico es un elemento central para vincular el servicio con el trabajo académico del curso. El trabajo en grupo ha sido cooperativo, requiriendo realizar aportaciones complementarias para alcanzar un objetivo común. Las entidades donde se ha realizado la experiencia consideran que la intervención de los estudiantes es de gran ayuda, a pesar de que ésta es limitada en el tiempo, contribuyendo a la mejora de los procesos formativos que llevan a cabo en ellas.

Por su parte, como puntos débiles de la experiencia encontramos que las necesidades sobre las que realizar el servicio han sido presentadas por las entidades sociales, por lo que no han podido ser descubiertas por los estudiantes. El servicio ha sido continuado, compuesto por tareas repetitivas cuya realización supone una moderada exigencia e implicación de los estudiantes. La participación ha sido delimitada durante la intervención, realizando aportaciones puntuales requeridas por los educadores de las entidades. Asimismo, los dinamismos organizativos no han formado parte del proyecto.

Tras el análisis de los datos obtenidos en los informes y el debate pedagógico realizado, observamos que los

estudiantes manifiestan su satisfacción respecto a las competencias

desarrolladas y los aprendizajes alcanzados durante el proyecto. En este sentido, destacar que valoran la intervención realizada por el bien que provoca la actividad en los receptores del servicio. Se han implicado personalmente y asumen el compromiso con la entidad social donde han realizado el servicio y actúan de manera reflexiva durante la intervención, aplicando las teorías de la asignatura relacionadas con la intervención.

En definitiva, la experiencia de ApS presentada favorece una ciudadanía crítica y comprometida con la mejora de vida de la comunidad, permitiendo la conexión de la institución universitaria con el tejido social próximo.

\section{Referencias bibliográficas}

Aramburuzabala, P. (2013).

Aprendizaje-servicio: una herramienta para educar desde y para la justicia social. Revista Internacional de Educación para la Justicia Social, 2(2), 5-11.

Arranz, P. (2011). La Universidad de Zaragoza en materia de Responsabilidad Social. En I. Saz (coord.). Contribución de las organizaciones a la consecución de los objetivos de desarrollo del milenio. 
Valencia: Tirant lo Blanch.

Grup de Recerca en Educació Moral (2014). Rúbrica para la autoevaluación y la mejora de los proyectos de ApS. Barcelona: Centre Promotor d'Aprenentatge Servei. Recuperado de http://www. aprenentatgeservei.org/intr a/aps/documents/aps autoevaluacio C ast IMP A5.pdf

Inciarte, A., Parra-Sandoval, M. C. y Bozo, A. J. (2010). Reconceptualización de la universidad. Una mirada desde América Latina. Maracaibo: Ediciones Astro Data.

Martínez, M. (2006). Formación para la ciudadanía y educación superior, Revista Iberoamerica de Educación, 42, 85-102.

Montes, R., Tapia, M. y Yaber, L. (2011). Manual para docentes y estudiantes solidarios. Buenos Aires:

CLAYSS. Recuperado de

http://www. clayss.org.ar/04 publicacio nes/Natura2013.pdf

Palos, J. (2015). Los proyectos de ApS siguen etapas bien establecidas y han de estar abiertos a cambios imprevistos. En J. Puig (coord.). 11

Ideas Clave. ¿Cómo realizar un proyecto de aprendizaje servicio? (103114). Barcelona: Graó.

Puig, J. M., Batlle, R., Bosch, C., y Palos. J. (2006). Aprenentatge Servei. Educar per a la ciutadania. Barcelona: Octaedro/Fundació Jaume Bofill.

Saz, I., Ramo, R. M. (2015).

Aproximación a los impactos y beneficios del aprendizaje servicio en la Universidad de Zaragoza. RIDAS,
Revista Iberoamericana de Aprendizaje Servicio, 1, 9-27.

Tapia, M.N. (2006). Aprendizaje y servicio solidario. Buenos Aires: Ciudad Nueva.

Tejada, J. (2013). La formación de las competencias profesionales a través del aprendizaje servicio. Cultura y Educación, 25(3), 285-294. 